\title{
TAILPIECE
}

\section{No Pain, No Gain. \\ Part II. A Personal Conceptualisation Of PTSD And Post Traumatic Psychological Difficulties}

\section{Palmer}

\section{Key Words}

PTSD. Debriefing. Grief. Bereavement. Loss. Classification. Media. Culture. Change. Anger.

There can be little doubt as to the psychosocio-cultural cost of combat for some soldiers. Those working in this area will, however, be confronted, from the outset, with inconsistencies between clinical care and management and published research. Biomedical researchers recruit motivated compliant subjects under the selection bias of strict inclusion and exclusion criteria. Clinicians treat distressed patients whose conditions seldom fulfil diagnostic criteria and who are often dealing with sociocultural issues and co-morbid conditions excluded by researchers. The generalisability from the study of subjects to the treatment and care of patients is not straightforward and is dependent upon numerous, generally uncontrollable, variables in patients. Physician related variables include experienced dissonance between clinical practice and research; levels of belief and confidence in research evidence and the strength of evidence upon which advice and guidelines may be based; the applicability of statistical results to individual cases; psychological mindedness; simple prejudice, habit and pressure of work.

Research may undermine the perceived benefits of experience that are generally unmeasurable and, therefore, considered of lesser merit. Clinical practice is an art, fraught with uncertainty and risk. Research is one path in the search for certainty and relief of anxiety, another is conformity to the group, but should research lead to proscriptive guidelines adopted by practitioners we run the risk of retarding progress for the short-term gain of lowered anxiety (and possible litigation) as did the followers of Galen.

\section{Col I Palmer L/RAMC}

Tri Service Professor of Defence Psychiatry

Royal Centre for

Defence Medicine,

Oak Tree Lane,

Selly Oak,

Birmingham, B29 6JF

Email:

ianpalmer@doctors.org.uk what can't be measured easily isn't important. That is blindness. The fourth step is to say that what can't be measured really doesn't exist. This is suicide. Post traumatic psycho-socio-cultural difficulties are multifactorial in aetiology and this leads, in my opinion to many of the difficulties in this area.

From the outset, I was beset with inconsistencies such as individuals with a 'full-blown' clinical picture of PTSD without the necessary 'qualifying' event and vice versa. Later a moment's introspection revealed to me that all the symptoms of PTSD could be seen after the break-up of a love affair, so was I dealing with a normal human experience or a mental disorder? I grew increasingly uncomfortable trying to put distressed individuals into the categorical 'boxes' required for a diagnosis of PTSD. Before long, I became almost antithetical to the label of PTSD, that I felt was becoming therapeutically stifling, until I started to think of psychological reactions to trauma in dimensional terms.

I realise, however, that such a suggestion may be counter to a Western medical orthodoxy based on measurement, embedded in a 'Culture of Blame', fed on voyeuristic media schadenfreude, to believe that all individuals will be 'damaged' or develop mental disorders following exposure to life's vicissitudes. Would it be radical to postulate that, whilst all individuals will be changed by their experiences, few are 'damaged' or develop mental disorders and some even benefit from it? That individuals and communities have more resources and abilities in dealing with life's stresses and strains than we, as a society, are led to believe?

\section{Post Traumatic Stress Reactions}

Without an aetiological basis for diagnoses, psychiatry applies a categorical approach. Whilst categorical classification systems have allowed advances in medicine, they are not without drawbacks in clinical practice and may, in my opinion, become reified in some quarters even medicalising what are, in essence, normal human emotional reactions and experiences. Categories are hypothetical constructs born of research diagnostic criteria, predominantly in the USA, and of particular relevance and value in the research and understanding of serious mental illness such as schizophrenia. Clinical practice 
seldom reveals the clear-cut diagnoses found in research. Since 1980 there has been much debate about whether or not PTSD has 'always existed'. As it was only 'defined' in that year, it cannot have 'existed' before.

However, examination of evidence from common human experiences such as the loss of personal relationships, language in common usage and grief, clearly reveals the ubiquity of the symptoms, and associated behaviours of PTSD; which suggests, to me at least, that our forbears knew all about them and that meaning is the key in their precipitation and perpetuation.

Firstly, the loss of personal relationships. After the break-up of a love affair, it is common to have intrusive thoughts of the individual that may be triggered by places or activities. Such triggers may be avoided in order to minimise distress and the associated arousal phenomena of anxiety, fitful sleep, dreaming and anger that may further exacerbate the situation. Associated behaviours such as turning to drink, finding another partner on the 'rebound', withdrawing from social contact etc. are experiences we all share. We generally survive with succour from friends and family until, eventually, the whole episode, and its attendant changes, is accepted, assimilated and accommodated; life goes on. The personal meaning of the loss is instrumental in the distress we bear. We learn from the experience which colours our subsequent relationships.

Secondly, language in common usage. That Society has been well aware of the post traumatic mental processes of re-experiencing, avoidance and arousal is reflected in common parlance viz. the statement that someone has something 'on their mind' (reexperiencing \pm arousal) furthermore, it is common experience that equilibrium is restored once the problem is solved. Individuals are commonly told not to 'dwell on problems' or to 'put them to the back of your minds' (avoidance) and we know what is meant when someone is 'wound up' (aroused).

Lastly, grief. There are similarities with the process of bereavement and grief, especially after combat where losses of various kinds are ubiquitous. As in grief, idiosyncratic meaning colours the process of acknowledgement, acceptance, assimilation and accommodation required to manage the changes created by the losses.

\section{The Grief Paradigm}

Bereavement is the ubiquitous human experience following loss through death. We all recognise that the experience is idiosyncratic and, whilst most cope, some develop problematic bereavement reactions and even mental disorder. I believe bereavement offers a valid simile for understanding and managing post traumatic stress reactions.

Loss is inherent in both grief and traumatic events. Both haul ontological issues into conscious awareness, disturbing faith in the continuity and predictability of life. Other losses following exposure to traumatic events include loss of: control; 'innocence'; faith; body image; trust in authority and selfesteem. It is my experience that healing after traumatic events and grief requires acknowledgement of the ontological issues of existence, the serendipitous and precarious nature of existence and our role in the process of 'being'.

Over our life cycle, our views about death change, life may even be seen by some as a preparation for death. For young children, it is a reversible phenomenon. For an adolescent, death happens to other people not them. For an octogenarian, it may not come soon enough. Unsurprisingly, combat soldiers are generally in the 17-25 year old group where existential omnipotence is at its zenith and magnified by group membership.

I believe that contemplation of the life cycle is necessary in any therapeutic intervention following exposure to traumatic events. Traumatic loss should be contextualised with reference to the individual's position in their life cycle in order to avoid the voyeurism and therapeutic nihilism of exclusive focus on the traumatic event(s).

\section{Change, Anger, Trust and Forgiveness}

Change is both inevitable and irrevocable following loss events. Daily, non-traumatic, life experiences have the ability to change our knowledge, understanding, character and behaviour. Thus, the oft-heard plea of 'if only ...', the fantasy of 'magical thinking' requires dispelling, from the outset.

The loss of control and helplessness created by major trauma leads to attempts to regain emotional control by withdrawing emotionally and/or socially. This is a manifestation of avoidance but only adds to an individual's problems by exacerbating interpersonal difficulties. It is usually allied to anger expressed either actively or passively.

Anger is common following both grief and traumatic events. In psychoanalytic terms, this may be understood as the introjection of a 'bad object', which, if accompanied with loss of 'good objects' (self), can lead to the psychological defences of 'identification with the aggressor', 'displacement' and 'projection' that manifest in 'acting-out' behaviours. Internalised anger may lead to depression and substance misuse. Externalised anger, both active and passive, can lead to blame, non-compliance or non-engagement in therapy. Should a soldier have been involved in killing, the attendant anger may be very frightening for an inexperienced therapist. If, however, the therapeutic relationship is to 
commence and develop in any meaningful way this anger or rage must be acknowledged and 'held', or contained, psychologically.

Projection often involves blame, which may be expressed through legal redress. Blame is counter-therapeutic. Other than bringing the perpetrators of human rights violations to justice, I know of little direct therapeutic benefit of legal action. The whole legal process of seeking compensation or the apportion of blame, whilst natural, is often long and tortuous and may be therapeutically damaging to individuals and their families, delaying the resolution of distress.

As trust may be undermined or lost following traumatic events, trust in the therapist is of paramount importance and requires honesty, continuity, steadfastness and robustness in its development and maintenance. Forgiveness is seldom mentioned but it is essential for any healing process as it alone can free us from the past. It involves confronting everyone involved, the survivor and perpetrator(s); which may include God. Forgiveness is about acceptance and resolution, not about excusing people or exonerating them from blame in 'extenuating circumstances'. Some may be unable to forgive, as they fear it may lead to forgetting and issues of 'Survivor Guilt' must be expressly addressed.

\section{The Aetiology of Post Traumatic Psychological Difficulties}

We are all products of our cultures, societies and private pasts, not tabula rasa. We thus bring all our cognitive, emotional, spiritual and physical strengths and weaknesses to any traumatic event. All events occur in an environment which contributes to the meaning and effect of the event both at the time and afterwards.

Psychological sequelae of trauma are the product of an interaction between the following, largely uncontrollable variables:

- The Individual. Strengths and vulnerabilities (genetic, physical, cognitive, emotional, social, cultural).

- The Event. Threat (through meaning), severity, duration.

- The Environment. Before, during and after.

- The Culture. The shared cultural values, mores and support.

Although all post traumatic stress reactions are the product of this interaction there is little doubt that the worse the event, in terms of being outwith an individual's possible experience, the more likely the psychological distress and difficulty. Environment is important, for if the trauma was experienced as a group this can provide integral psychosocial support for its members. If an individual has poor interpersonal relationships or a multitude of problems before encountering trauma, the experience can only add to their stock of subsequent problems. Cultural events, such as funerals, are important in helping individuals process their experience. The intrusive and speculative intrusion of Western media culture may interfere with societal, or individual attempts, to assimilate and accommodate the changes wrought by trauma.

The genesis of post traumatic reactions and disorders is thus complex and multifactorial, as is its management by individuals, either alone or with help. Time is said to be the great healer; this is not always true. Nevertheless, there is concern that early psychological interventions such as psychological debriefing may actually interfere with the initial processing of an experience and create later problems.

Military debriefing occurs in all Armies after any mission, so lessons may be learned and mistakes avoided. In WWII a number of 'historical' research debriefs on the battlefield following combat were noted to be therapeutic for some soldiers and from WWI onwards, the psychosocial interventions, PIES \& $7 \mathrm{Rs}^{\star[1]}$ were found to be effective following acute combat breakdown. Naturally attempts were considered to use such interventions with individuals who had or did not define themselves as suffering from any break down in the hope of preventing PTSD.

I used to be Jesuitical in my belief in psychological debriefing. Having undertaken it in the field, my assumption that soldiers would welcome such intervention, and even benefit from it, was naïve given the anger generated within such groups. Furthermore, given the multifactorial genesis of post trauma psychological difficulties, it is difficult to believe that such interventions could prevent post traumatic mental disorders. I believe they may, however, be of some use; if the timing is right, and here I am guided by examination of the grieving process in which the initial psychic 'shock' makes it difficult and undesirable to talk as the reality of the loss is assimilated. To be coerced to attend a group or talk within 72 hours is highly likely to exacerbate the anger and arousal of acute grief and disrupt the healing process. However, there usually is a time when individuals wish to talk, the timing of which will vary from person to person and will initially involve family, friends and colleagues. Only if this is not helpful, or the distress is unmanageable should individuals seek professional help.

I believe grief offers a template for the early management of post traumatic stress

\footnotetext{
${ }^{\star}{ }^{[1]}$ PIES [Proximity, Immediacy, Expectancy, Simplicity] 7R’s [Recognition, Respite, Rest, Recall, Reassurance, Rehabilitation, Return (to duty)].
} 
reactions. The first step is the human one of reaching out and acknowledging the individual's distress and suffering and offering whatever help is acceptable to the individual - expecting it to be declined. The next step is to provide an unobtrusive 'watching brief' whilst maintaining the offer of help and support, acknowledging that time must pass before the loss is accepted and changes assimilated and accommodated. The aim is to provide help at a time of the individual's choosing, which may be delayed. Remember, whilst there may be problems for some, most people will settle without professional intervention and that processing is achieved when memories may be accessed without attendant disruptive emotions. With luck, any good within the experience has also been accepted. I would thus like to see psychological reactions to trauma accepted and managed in a way similar to grief and bereavement.

\section{My Practice}

Within society there is a debate, a negotiation if you will, between interested parties survivors, lawyers, politicians, doctors and therapists about the subject. Psychotherapy is expensive in time and personal and fiscal costs. Even the most physical of psychiatrists in WWI, Mott, felt that it required a considerable degree of training and demanded "a great deal of time and individual attention" in addition he noted "we have to accept that listening to the tragedy of war experience is a painful business for the therapist." And he believed that "the people who can touch psychic wounds with delicacy and sympathy are extremely rare, especially amongst doctors..”. I think this remains true today.

My psychotherapeutic approach is eclectic. For me, traumatic events are about loss of good objects and introjection of bad ones; integral to which are both loss, anger and perhaps at times rage. All of my patients have required 'holding' or 'containing' in some way. I believe that if I cannot 'hold' a patient's anguish, pain and rage I cannot expect them to do so. Furthermore, to show my fear will only reinforce their feelings of isolation, loathing and anger.

I have found that rapid control of symptoms through a mixture of cognitive, behavioural and pharmacological means are vital to restore an individual's feeling of control. However, in my opinion work is always required on the meaning of the event if healing is to take place.

I accept everyone is changed by their experience of traumatic events but I challenge the current societal view that such change is always negative. Traumatic events (and grief) remind us we shall all die and must make our peace with this fact. This may be the lesson to be learnt from exposure to unpleasant events. To be free of the fear of death makes life more immediate, real and fulfilling.

\section{Conclusion}

In these pieces, I have adumbrated my understanding of the psycho-socio-cultural aspects of soldier's experience of combat drawn from 27 years as an Army Medical Officer and 13 years as an Army Psychiatrist. During my time in service I have come to learn the importance of history in this understanding.

No military officer has ever been commissioned without detailed study of Military History. No military psychiatrist should ever practice without thorough study of the History of Military Psychiatry. They are likely, as was I, to be humbled to find they have much in common with their psychiatric ancestors. I was pleased to find that my clinical practice and understanding had already been described by W H R Rivers who, in 1917, recommended that "instead of advising repression and assisting it by drugs, suggestion, or hypnotism, we should leave the patient to resolutely face the situation provided by his painful experience. We should point out to him that such experience... can never be thrust wholly out of his life ... his experience should be talked over in all its bearings. Its good side should be emphasised, for it is characteristic of the painful experience of warfare that it usually has a good or even noble side, which in his condition of misery the patient does not see at all, or greatly underestimates."

Army Psychiatry teaches the importance of an individual's culture on the genesis and prevention of mental disorder. Discourses on soldiers' experiences have led to understanding about individual and collective traumatic memories, the unconscious and how society negotiates its slow accommodation and assimilation of what is 'normal' psychologically following trauma. Whilst accepting neuro-humoural explanations may underlie post traumatic mental disorders such as PTSD, I believe the psycho-sociocultural to be more important in these negotiations.

Bereavement, traumatic events and the media, serve to remind us of the arbitrary and serendipitous nature of existence and being. Medical 'science' may offer potential answers but can we put our faith in science, which, after all, is based on falsifiable hypotheses. Medicine can extemporise on the all too visible pain of individuals and communities relayed daily in the media and even be called upon for professional opinion when redress or compensation is sought; but how many health workers contemplate the social consequences of their diagnoses and pronouncements in a society where the media feed on the carrion of human misfortune. The media fuel and feed our appetites for schadenfreude, voyeurism and the unusual. News is news by dint of the fact that the events reported are rare or unusual and may be part of history in the making. Such events are outside many of our 
experiences and, therefore, of interest to us. Being involved in a traumatic event or its aftermath may offer the possibility of being part of history. Before we get involved in helping those traumatised we must examine our motives. We may genuinely care or need to vicariously work through our own needs. Whatever the reason, we get involved for a reason. But do we need to and if so when?

I have latterly found more interesting discourse in the writings of (military) psychiatric historians than of current academic psychiatric publications and agree with Feudtner who states "whilst PTSD provides a language with which to acknowledge and describe the impact of horrible events on people's lives, it always groups so many diverse experiences together in the name of medical science that it strips them of their specific meaning and consequence. And this may ultimately prove to be not only deductively dehumanising but also intellectually and therapeutically stifling".

Trauma is at the very heart of human existence and teaches us much about it. We learn from our military forbearers about psychological limits and boundaries, strengths and weaknesses, survival, endurance, determination and perseverance. Of altruism and despotism, heroism and cowardice.

My career in the Army has humbled and privileged me by allowing me direct experience of traumatic events, academic time to study the subject and most importantly the honour of trust by those who have seen fit to tell me their stories.

No pain, no gain. 